\title{
SPECIAL INTEREST GROUPS
}

\section{South African Society of Physiotherapy}

obstetric Association:

Secretary: Mrs. B. Kastell,

11 Bath Avenue,

Parkwood,

Johannesburg,

2193.

Tel.: 42-7410.

Manipulative Therapists Group:

Secretary: Miss C. de Smidt,

Erin Villa,

5 Lower Trill Road,

Observatory,

7925.

6.A. Neurodevelopmental Therapy Association:

Secretary: Mrs. L. Freeling,

P.O. Box 792 ,

Krugersdorp,

1740.

\section{Hospital Group:}

Secretary: Miss Pat Bowerbank,

605 Tivoli Flats,

9th Avenue,

Wonderboom South,

Pretoria,

0084

Private Practitioners' Association:

Secretary: Mrs. A. Miot,

P.O. Box 6681 ,

Johannesburg.

Phone 784-3328.

\section{Lecturers Group:}

Secretary: Miss S. Irwin-Carruthers, Physiotherapy Department, P.O. Box 63,

Tygerberg,

7505 .

(continued from page 10)-Influence on the effectiveness of coughing:

\section{References}

7. Mead, J. (1967): Significance of the relationship between lung recoil and maximum expiratory flow, J. Appl. Physiol., 22 (1), 95-108.

8. Don, H. F., Wahba, W. M., Craig, D. B. (1972): Airway closure, gas trapping and the Functional Residual Capacity during Anaesthesia, Anesthesiology, 36, 6, 533 .

9. Gaskell, D. V., Webber, B. A. (1973): The Brompton Hospital Guide to Chest Physiotherapy, 2nd. ed., Great Britain, Blackwell Scientific Publications, pp. 10,60 .

10. Cash, Joan E. (1975): Chest, Heart and Vascular disorders for Physiotherapists, London, Faber and Faber, pp. 90, 198.

11. Articles for the SAMJ (1975): S. Afr. Med. J., 49, 2099.

\section{ABSTRACTS}

Gault, W. R. \& Gatens, P. F.: Use of Low Intensity Direct Current in Management of Ischemic Skin Ulcers. Physical Therapy 56 (3): 265-269, March 1976.

A clinical investigation of the use of low intensity direct current (LIDC) in treating ischemic skin ulcers was conducted at the University of Missouri. The purpose of the study was to evaluate the effect of LIDC on altering the healing rate of ischemic skin ulcers. One hundred ulcers were treated with LIDC. Six of the patients had bilateral symmetrical ulcers which provided the control group. The six control ulcers were managed exactly the same as their counterpart except that LIDC was omitted. The mean healing ratio of the control ulcers was 14,7 per cent per week compared to 30 per cent per week of the treated counterpart. The mean healing rate of the 100 ischemic skin ulcers treated with LIDC was 28,4 per cent per week. The data indicated that ischemic skin ulcers treated with the current responded with a healing rate approximately twice as fast as those ulcers that did not receive LIDC treatment. The authors concluded that the use of LIDC added an effective modality to the armamentarium of the physical therapist for the treatment of ischemic skin ulcers.

Authors' Summary.

Weisberg, J.: Influence of icing and brushing on the Achilles' tendon reflex in adult human subjects. Physiotherapy Canada 28 (1): 21-23, March 1976.

The technique of icing and brushing the skin overlying a muscle group, as originally developed by Margaret Rood, was used over the gastrocnemius in this study, to determine whether an alteration in amplitude of the Archilles' tendon reflex is produced.

Two groups of healthy adult human subjects aged between 14 and 40 years - an experimental group (16 subjects) and a control group (15 subjects) - were tested. Both legs of each subject in the test group were stimulated, after one side was "treated" for 20-25 seconds with brushing and then for 5-10 seconds with icing. The subsequent foot displacement was measured with a photoelectric device, and the amplitude of the free Achilles' tendon reflex was recorded on an electrocardiogram.

The results of the study showed a mean increase in amplitude of the Achilles' tendon reflex of $40 \%$ on the "treated" side (10\% on the "untreated" side) in the experimental group. The author therefore concludes that icing and brushing over the muscle does bias the muscle spindle, enhancing muscular contraction.

J.M.

Zimnicki, B. A. \& Fernie, G. R.: Biofeedback and the lower extremity amputee: a new training aid. Physiotherapy Canada 28 (2): 79-82, May 1976.

A simple biofeedback device has been developed to aid in the prosthetic training of above-knee geriatric amputees. It consists of a switch which when attached to the prosthetic knee joint causes a buzzer to sound whenever the joint is not fully extended. A training protocol has been devised and has been used with success on nine patients who had difficulty in learning. The biofeedback trainer both focuses the attention of the patient on knee control and provides a more immediate and simple signal of errors than can be provided by a therapist. This system can be used by the patient in the absence of the therapist thus making better use of his hospital stay.

Authors' Summary. 